\title{
TWO-COLOR TWO-PHOTON IONIZATION SPECTRUM OF PYRENE IN N-HEXANE
}

\author{
KEIJI NAKASHIMA*, HIROFUMI SOGA \\ and TEIICHIRO OGAWA \\ Department of Molecular Science and Technology, Kyushu \\ University, Kasuga, Fukuoka 816, Japan
}

(Received 13 June 1996)

The photoionization process of pyrene in non-polar solvent has been investigated using a two-color two-photon ionization technique. The ionization spectrum near threshold of pyrene in $n$-hexane was measured by a delayed irradiation of a dye laser $20 \mathrm{~ns}$ after an $\mathrm{N}_{2}$ laser at total excitation energies of 5.9-6.22 eV. The two-photon ionization threshold of pyrene was in the vicinity of $5.9 \mathrm{eV}$ which is larger by $0.7 \mathrm{eV}$ than that expected from the onset of one-photon ionization. The ionization spectrum shows maxima at 5.92 and 5.99 $\mathrm{eV}$ which may be assigned to an autoionization process.

Keywords: Multiphoton ionization; two-color ionization; pyrene; non-polar solvent; autoionization; ionization threshold

\section{INTRODUCTION}

Investigation of photoionization process of molecules in non-polar solvents provides interesting information on the interaction between solute and solvent. Although the interaction of a neutral solute with non-polar solvent is weak, the ionization of solute gives rise to strong coulombic force, which remarkably decreases the ionization potential of solute [1-4]. The photoionization threshold in solution, $I P_{L}$, is expressed with the polarization energy of the cation, $P_{+}$, and the electron affinity of solvent, $V_{0}$ :

$$
I P_{L}=I P_{G}+P_{+}+V_{0}
$$

*To whom correspondence is to be sent. 
where $I P_{G}$ is the gas-phase ionization potential. $P_{+}$is negative and of the order of 1-2 eV, depending mainly on molecular size of the cation and the dielectric constant of the solvent [5]. $V_{0}$ ranges from -0.6 to +0.1 $\mathrm{eV}$ for saturated hydrocarbon solvent, depending on the solvent [6-8].

Laser multiphoton ionization is useful in detecting a weak ionization signal because it has an advantage in sensitivity due to the use of a strong light source and tightly focused light enables the signal to increase even at low laser power [9-11]. Mechanism of the resonance enhanced multiphoton ionization (REMPI) can be probed by the two-color ionization method where one laser is used for the excitation to the intermediate state and the second laser for ionization $[1,2,11]$. Hoffman and Albrecht have measured weak ionization signal of $\mathrm{N}, \mathrm{N}, \mathrm{N}^{\prime}, \mathrm{N}^{\prime}$, -tetramethylphenylenediamine near the threshold by means of the two-color method [3].

In this work, we have measured the two-photon ionization spectrum of pyrene $\left(\mathrm{C}_{16} \mathrm{H}_{10}\right)$ in $n$-hexane. Two dissimilar ionization thresholds have been reported for pyrene in non-polar solvents. Holroyd et al. [1] reported a threshold of $6.20 \mathrm{eV}$ for one-photon ionization of pyrene in 2 , 2-dimethylpropane (neopentane). Siomos and Christophorou [9] reported a much lower threshold of $4.80 \mathrm{eV}$ for one-color multiphoton ionization in $n$-pentane. This large discrepancy in the two experiments has not been clearly explained, yet. In our previous work [11], we measured a photoconductivity of pyrene in $n$-hexane by means of the concerted irradiation of the second harmonics of an Nd-YAG laser and a nitrogen laser (total excitation energy: 5.65 and $6.01 \mathrm{eV}$ ), and found photoionization occurs at these energies. In the present work, we have measured the two-color ionization spectrum of pyrene using a nitrogen laser for the excitation of the intermediate state and a nitrogen-laserpumped dye laser for ionization:

$$
\begin{aligned}
& \mathrm{C}_{16} \mathrm{H}_{10}+h v_{1}(337 \mathrm{~nm}) \rightarrow S_{2} \rightarrow S_{1} \\
& S_{1}+h v_{2} \text { (visible) } \rightarrow \mathrm{C}_{16} \mathrm{H}_{10}+e^{-},
\end{aligned}
$$

in the energy region of 5.9-6.22 $\mathrm{eV}$ which is near the threshold of one-photon ionization. We have obtained the photoionization threshold of pyrene with highly sensitive method and verified the mechanism of the photoconductivity of pyrene at low energy. 


\section{EXPERIMENTAL}

The apparatus used in this work is similar to that used in the previous work [11] except for the use of a dye laser for ionization. In brief, a small part of $337 \mathrm{~nm}$ light from an $\mathrm{N}_{2}$ laser (Molectron UV24, $3 \mathrm{~mJ} /$ pulse, 10 ns (FWHM)) was introduced into an ionization cell to excite the sample. The visible light from a dye laser pumped by the $\mathrm{N}_{2}$ laser (Molectron DL14P) was introduced into the cell from the opposite direction at $20 \mathrm{~ns}$ after the irradiation of the $\mathrm{N}_{2}$ laser. The dye laser beam was focused with an achromatic lens (focal length $50 \mathrm{~cm}$ ) and the $\mathrm{N}_{2}$ laser beam only passed through a $2 \mathrm{~mm}$ diameter pinhole without focusing for the two beams to be well-overlapped by each other. The visible beam was passed through an optical delay line in order to adjust its irradiation timing to $20 \mathrm{~ns}$ after the irradiation of the $\mathrm{N}_{2}$ laser. Three different laser dyes were used to cover the region of $428-475 \mathrm{~nm}$ : cumarine-440/ethanol for 428-448 nm, cumarine-460/ethanol for $444-470 \mathrm{~nm}$, and cumarine$481 / \mathrm{p}$-dioxane for $465-475 \mathrm{~nm}$. The intensities of two lasers were monitored with two photodiodes, respectively. The wavelength dependence of the sensitivity of the photodiodes was calibrated with a power meter (Molectron Power Max 5100).

The ionization cell consists of a quartz fluorescence cell and a pair of copper electrodes: electrode spacing was $3 \mathrm{~mm}$. One of the electrodes was applied an bias voltage of $2.5 \mathrm{kV}$ and the transient current induced by the laser irradiation was measured through the other electrode with a current amplifier (Keithley Model 427), a digital oscilloscope (Iwastsu DS-6411, $40 \mathrm{MHz}$ ), and a microcomputer (NEC PC9801VF2).

Pyrene (Wako Chemical, research grade) was purified by vacuum sublimation. Hexane (Wako Chemical, research grade) was distilled and dried with $\mathrm{CaCl}_{2}$. The sample solution was bubbled with $\mathrm{N}_{2}$ gas for 5 minutes so as to remove oxygen. The experiments were carried out under room temperature.

\section{RESULTS AND DISCUSSION}

Typical time profiles of the transient current from pyrene in $n$-hexane $\left(1 \times 10^{-5} \mathrm{~mol} / \mathrm{dm}^{3}\right)$ produced in the two-color ionization using the dye laser and the nitrogen laser pulse, and those in the one-color ionization 
using each laser are shown in Figure 1. The time resolution of current measurement was $10 \mathrm{~ms}$. The laser pulse energies were $2 \mu \mathrm{J} /$ pulse and $200 \mu \mathrm{J} /$ pulse for the $337 \mathrm{~nm}$ and visible light, respectively. Since the one-color ionization by the dye laser requires three photons, one-color irradiation of the visible light gave rise to negligible signal compared to the two-color signal. The intense $337 \mathrm{~nm}$ light induces strong photocurrent due to REMPI and, therefore, the intensity of $\mathrm{N}_{2}$ laser was kept small enough to restrict the one-color two-photon ionization. In our experimental condition, the photocurrent produced by the two-color irradiation of $337 \mathrm{~nm}$ and visible lights, Q(337+VIS), were more than five times as large as the one-color signal due to the nitrogen laser, $\mathrm{Q}(337)$. In all measurement shown in Figure 1, no photocurrent was observed by the irradiation to the solvent without solute. Thus ionization of $n$-hexane and its impurity was negligible.

The net signal due to two-color ionization, $\Delta \mathrm{Q}$, was obtained as the difference of $\mathrm{Q}(337$ + VIS) and $\mathrm{Q}(337)$ as,

$$
\Delta \mathrm{Q}=\mathrm{Q}(337+\mathrm{VIS})-\mathrm{Q}(337)
$$

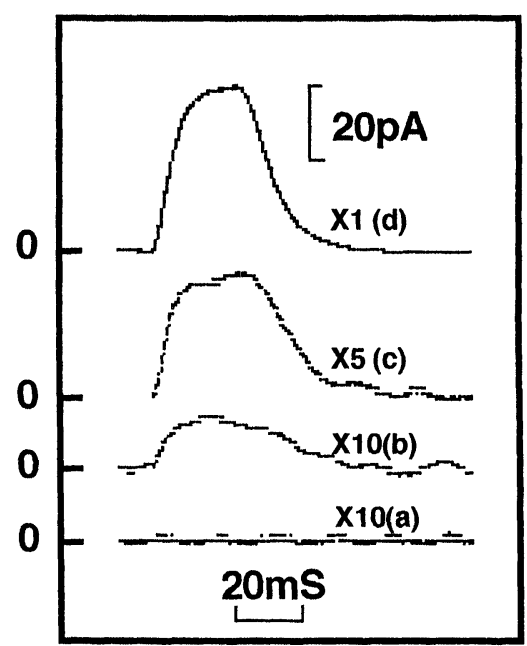

FIGURE 1 Transient current induced by the irradiation of laser pulses on pyrene in cyclohexane. (a) $460 \mathrm{~nm}$ light only ( $200 \mu \mathrm{J} /$ pulse), (b) $337 \mathrm{~nm}$ only ( $2 \mu \mathrm{J} /$ pulse), (c) $470 \mathrm{~nm}$ with $337 \mathrm{~nm}$, and (d) $460 \mathrm{~nm}(150 \mu \mathrm{J} /$ pulse $)$ with $337 \mathrm{~nm}(2 \mu \mathrm{J} /$ pulse $)$. 
Figure 2 (a) and (b) shows the dependence of $\Delta \mathrm{Q}$ on the intensities of the $\mathrm{N}_{2}$ and the dye lasers, respectively. The transient current was linearly proportional to the intensity of the $\mathrm{N}_{2}$ laser at every dye-laser wavelength. The two-color signal was linearly proportional to the laser intensity below $470 \mathrm{~nm}$, and rapidly became smaller at longer excitation wavelengths and disappeared at about $475 \mathrm{~nm}$. Although $337 \mathrm{~nm}$ irradiation of pyrene initially produces the $S_{2}$ state [12], the delay time between the two lasers ( $20 \mathrm{~ns}$ ) is long enough for the $S_{2}$ state to relax to the $S_{1}$ state. Therefore, this result indicates that the two-color signal is produced by $1+1$ REMPI via the $S_{1}$ state at the day laser wavelength below $475 \mathrm{~nm}$. The total excitation energy is the sum of the energy level of the $S_{1}$ state $(3.32 \mathrm{eV})$ and the photon energies of the visible light. A very weak photocurrent was observed even above $475 \mathrm{~nm}$ at high laser fluence which is obtained using a shorter focus lens, but it had quadratic dependence on the dye laser intensity and may be assigned to
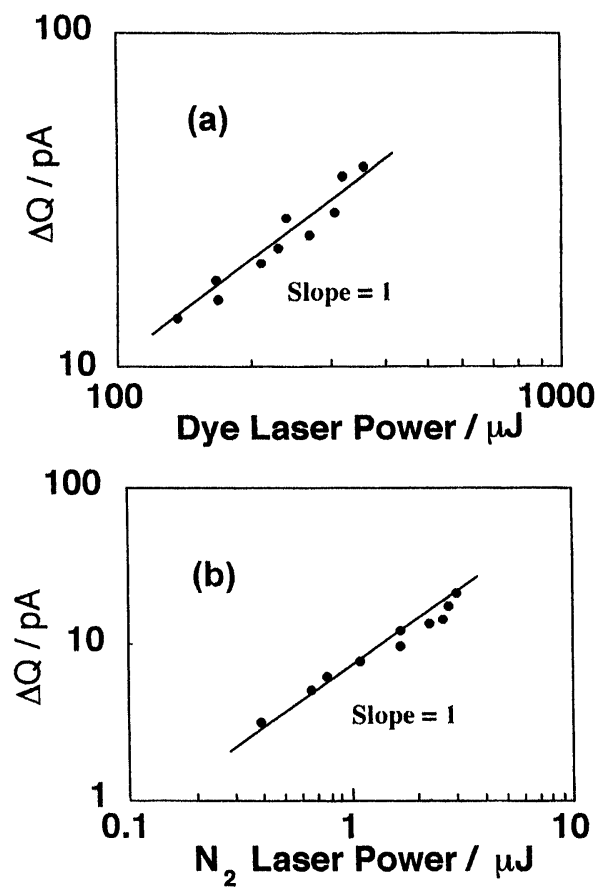

FIGURE 2 The dependence of the two-color enhanced signal on the pulse energies of (a) the dye laser and (b) the $\mathrm{N}_{2}$ laser. 
$1+2$ REMPI. Thus we measured the ionization spectrum only below $475 \mathrm{~nm}$.

The dependence of two-color ionization signal on the wavelength of dye laser for $428-475 \mathrm{~nm}$, i.e., the ionization spectrum of the $S_{1}$ state of pyrene, is shown in Figure 3 (a); the total excitation energy ranges from 5.9 to $6.22 \mathrm{eV}$ as the sum of the $S_{1}-S_{0}$ energy gap and the photon energies of the visible light. The ionization intensity was measured by dividing observed $\Delta \mathrm{Q}$ by the visible light intensity. The ionization spectrum has a maximum in the vicinity of $450 \mathrm{~nm}$ and disappeared at about $475 \mathrm{~nm}$, and this structure of the spectrum will be discussed later. The onset at $475 \mathrm{~nm}$ means that the threshold energy of pyrene in $n$-hexane should be about $5.9 \mathrm{eV}$. The potential energy due to the electric field used in our experiments $(8.3 \mathrm{kV} / \mathrm{cm})$ is less than $10^{-2} \mathrm{eV}$ at the thermalization radius of the photoejected electron in hydrocarbon solvent (about $10 \mathrm{~nm}$ [13]), and is negligible compared with the thermal energy at room temperature $(25 \mathrm{meV})$. Thus it should not be necessary to consider the electric field effect on the ionization threshold. From $V_{0}$ of

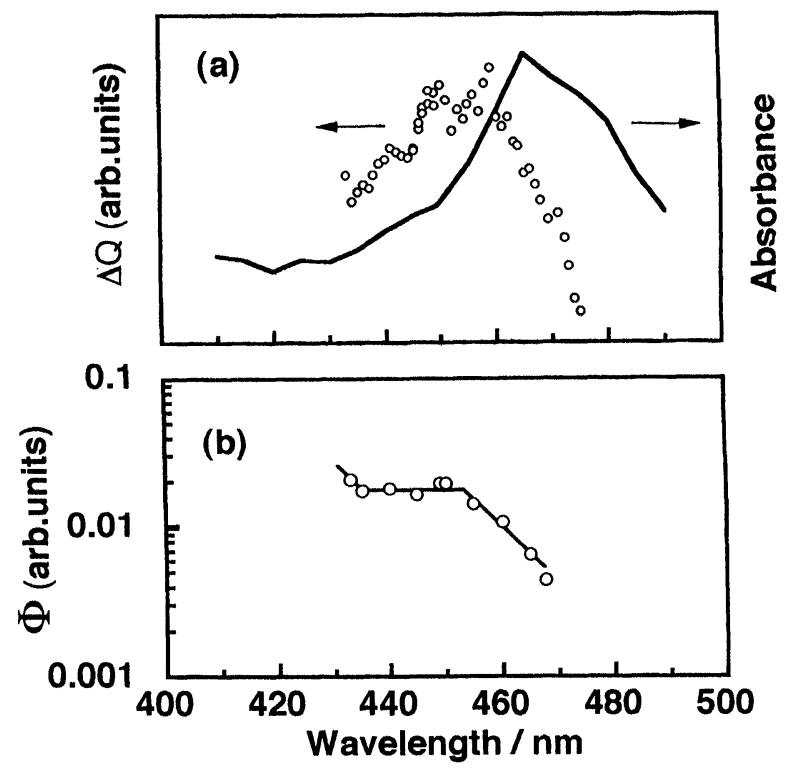

FIGURE 3 (a) open circles: Ionization spectrum of the $S_{1}$ state of pyrene, line: transient absorption spectrum of the $S_{1}$ state of pyrene. (b) Wavelength dependence of the ionization efficiency. 
$n$-hexane $(+0.04$ to $+0.09 \mathrm{eV}[6-8])$ and the adiabatic $I P_{G}$ of pyrene $(7.44$ $\mathrm{eV}[14]), P_{+}$in $n$-hexane is estimated to be about $-1.6 \mathrm{eV}$ according to Equation (1). Holroyd et al. reported the adiabatic threshold energy for one-photon ionization of pyrene in 2,2-dimethylpropane $\left(V_{0}=-0.43\right.$ $\mathrm{eV}[6])$ to be $6.20 \mathrm{eV}$ [1]. This corresponds to $P_{+}=-0.81 \mathrm{eV}$, which is in good agreement with the value expected from the radius of pyrene. Since the polarization energy of cation is essentially determined by the dielectric constant $(\varepsilon)$ of solvent and the cation radius $\left(r_{+}\right)$as [5]

$$
P_{+}=\frac{1}{2 r_{+}}\left(1-\frac{1}{\varepsilon}\right),
$$

$P_{+}$for $n$-hexane $(\varepsilon=1.88)$ should be nearly the same as that for $2,2-$ dimethylpropane $(\varepsilon=1.87)$. Nevertheless our result for $P_{+}$is smaller by $0.8 \mathrm{eV}$ than theirs; the adiabatic ionization threshold of pyrene in $n$-hexane using $P_{+}=-0.81 \mathrm{eV}$ is expected to be $\sim 6.6 \mathrm{eV}$ according to Equation (1). An unusually low threshold $(4.8 \mathrm{eV})$ was reported for pyrene in $n$-pentane by the one-color two-photon ionization method [9]. We could not observe any $1+1$ REMPI signal at lower energies than 5.9 $\mathrm{eV}$, but only $1+2$ REMPI signal was observed in this region as mentioned above. Thus no threshold at $4.8 \mathrm{eV}$ was observed in the present study. The ionization signal remarkably decreases near the threshold. When the photon density of the laser beam is intensified so as to obtain stronger two-photon signal, the three-photon ionization will increase in intensity and may exceed the photocurrent with the twophoton ionization and make a threshold unclear.

Considering the large difference between threshold energies of onephoton ionization [1] and the present study, we have firstly considered that the two-photon ionization threshold might come from some impurities. Since no photocurrent was observed for the irradiation of the solvent, a low-IP impurity contained in the solvent is not the origin of photocurrent. An impurity contained in pyrene may cause photocurrent. We have measured ionization spectra using two kinds of pyrene samples: one was sublimated only once and another was done three times. They agreed well within our experimental error $(10 \%)$. Although all the impurities cannot be removed completely by means of purification, it should change the ratio of impurities in pyrene. Thus we have concluded that the ionization threshold observed in the present work does not 
come from the low-IP impurity in pyrene. Another origin may be the photoinduced elecron transfer to some electron acceptor (A) involved in solvents as a contamination:

$$
\mathrm{C}_{16} \mathrm{H}_{10}+\mathrm{A}+2 h v \rightarrow \mathrm{C}_{16} \mathrm{H}_{10}{ }^{+}+\mathrm{A}^{-}
$$

The most probable origin is a small amount of oxygen solved in the solution. For verification, we have compared the spectra for the degassed and airsaturated solutions. However, no obvious difference was observed between them. Thus photoconductivity due to the electron transfer to an acceptor should not be the origin of the low-IP ionization. From these results, the photocurrent onset observed in the present work was found to be correctly the two-photon ionization threshold of pyrene.

In our previous work, we measured a ionization of pyrene in cyclohexane by means of $1+1$ REMPI at a fixed wavelength using the second harmonics of a Nd-YAG laser $(532 \mathrm{~nm})$ and a nitrogen laser [11]. According to that result, a weak photoconductivity is observed in the $1+1$ ionization via the $S_{1}$ state (total excitation energy: $5.65 \mathrm{eV}$ ), and, however, that via the $S_{2}$ state $(6.01 \mathrm{eV})$ was more intense. We could not clearly explain the preferential yield of the photocurrent via the $S_{2}$ state in the previous work. Since both lasers have pulse widths of 5-10 ns, the process via a short-lived intermediate state such as the $S_{2}$ state should be disadvantageous to ionization, and no strong photocurrent would be expected. However, this phenomenon is understandable if the ionization threshold of pyrene in $n$-hexane also lies in the vicinity of $5.9 \mathrm{eV}$. The energy of the ionization via the $S_{1}$ state $(5.65 \mathrm{eV})$ is slightly lower than $I P_{\mathrm{L}}$, and the photoconductivity at this energy should not result from direct excitation to the ionization continuum but from a "hot band" or a thermal dissociation of the geminate ion-pair which does not have enough energy to dissociate directly [8]. Thus the ionization via the $S_{2}$ state seems strong compared to that via the $S_{1}$ state because the energy level of the former $(6.01 \mathrm{eV})$ is above the ionization potential $(5.9 \mathrm{eV})$.

Siomos et al. have measured the fluorescence quenching of pyrene in $n$-hexane by an electron acceptor (perfluoro- $n$-hexane) and found that there is a weak onset of the ion formation at $5.99 \pm 0.03 \mathrm{eV}$, and another strong onset at $6.4-6.5 \mathrm{eV}$ [15]. The former should correspond to the threshold reported in this work and the latter to that for the one-photon ionization reported by Holroyd et al. [9]. Their measurement for pyrene 
was terminated at about $6.20 \mathrm{eV}$ and the existence of a weak photocurrent at lower energies might not be detected in their work. The weak ionization corresponding to the lower threshold can be detected only by the use of strong pulse lasers due to its weak intensity.

Now we turn our attention to the structure in the ionization spectrum, i.e., the maximum at $450 \mathrm{~nm}$. In the case of direct ionization, the photocurrent monotonously increases with excitation energy because the final state of ionization is continuous. Thus this existence of a maximum should be assigned to the autoionization of a neutral excitedstate, which is excited at specific energies $[4,16]$.

The relative ionization yield, $\phi=\Delta Q / \varepsilon^{\prime}(\lambda)$, is the ratio of the ionized molecules to the absorbed photons and was shown in Figure 3(b); $\varepsilon^{\prime}(\lambda)$ is the transient absorption coefficient of the $S_{1}$ state. $\phi$ rapidly decreases with wavelength above $450 \mathrm{~nm}$ and was constant for $430-450 \mathrm{~nm}$. In the condensed phase, the vibration of the photoexcited state initially relaxes after the excitation, and autoionization should mainly proceed through the vibrational ground state of an excited state $\left(S_{n}\right)$ as follows.

$$
\begin{gathered}
S_{1}+h v_{2} \rightarrow S_{n}\left(v^{\prime}\right) \\
S_{n}\left(v^{\prime}\right) \rightarrow S_{n}\left(v^{\prime}=0\right) \\
S_{n}\left(v^{\prime}=0\right) \rightarrow \mathrm{C}_{16} \mathrm{H}_{10}{ }^{+}+\mathrm{e}^{-}
\end{gathered}
$$

The energy dependence of the photoclirrent produced in this process is expressed as

$$
Q \propto \varepsilon^{\prime}(\lambda) \phi^{r}\left(v^{\prime}\right) \phi^{+}
$$

where $\phi^{r}\left(v^{\prime}\right)$ is the quantum yield of the vibrational relaxation (process (3)), and $\phi^{+}$is a quantum yield for the autoionization of the $S_{n}\left(v^{\prime}=0\right)$ state (process (4)). Consequently, $Q / \varepsilon^{\prime}(h v)$ should remain constant when $\phi^{r}\left(v^{\prime}\right)$ is constant over the vibrational band of the $S_{n}$ state.

No direct measurement of $\phi^{r}\left(v^{\prime}\right)$ for pyrene seems to be made. However, the fluorescence yield of pyrene in non-polar solvent is nearly constant around $6 \mathrm{eV}[16]$. The fluorescence yield is the product of $\phi^{r}\left(v^{\prime}\right)$ and the efficiency of the internal conversion from the vibrationally ground state of the $S_{n}$ state to the $S_{1}$ state; the latter is independent of the 
excitation energy for a single state. Thus $\phi^{r}\left(v^{\prime}\right)$ should be constant in this region. From these considerations the maximum in the ionization spectrum should be assigned to the autoionization of an excited state $\left(S_{n}\right)$. In the case of solid anthracene, an autoionization process has been observed below the threshold of the direct ionization, and its quantum efficiency is constant over the absorption of a single excited state [17].

Masuhara et al. reported the transient absorption spectrum of the $S_{1}$ state of pyrene and it has a maximum at $450 \mathrm{~nm}$ [18], which corresponds to the total excitation energy of $5.99 \mathrm{eV}$. This excited state should be identical with that observed in the absorption spectrum of pyrene in $n$-pentane at $5.99 \mathrm{eV}[14]$, and in the multiphoton ionization spectrum at $6.00 \mathrm{eV}$ [9]. The autoionizing state observed in this work, however, has a maximum at $450-460 \mathrm{~nm}\left(6.02-6.08 \mathrm{eV}\right.$ including $3.32 \mathrm{eV}$ of the $S_{1}$ state), and no maximum was observed around $465 \mathrm{~nm}(5.99 \mathrm{eV})$. Thus the autoionization peak seen in the two-color ionization should come from an excited state $\left(S_{n}\right)$ of weak absorption which is not clearly seen in the transient absorption spectrum of pyrene.

\section{References}

[1] Holroyd, R. A., Press, J. M., Böttcher, E. H. and Schmidt, E. H. (1984). J. Phys. Chem., 88, 744.

[2] Siomos, K., Kourouklis, G. A. and Christophorou, L. G. (1981). Radiat. Phys. Chem., 17, 75.

[3] Hoffman, G. J. and Albrecht, A. C. (1990). J. Phys. Chem., 94, 4455.

[4] Lee, K. L. and Lipsky, S. (1985). J. Chem. Phys., 82, 3650.

[5] Born, M. (1920). Z. Phys., 1, 45.

[6] Holroyd, R. A. and Allen, M. (1971). J. Chem. Phys., 54, 5014.

[7] Holroyd, R. A., Dietrich, B. K. and Schwarz, H. A. (1972). J. Phys. Chem., 76, 3794.

[8] Holroyd, R. A. and Russel, R. L. (1974). J. Phys. Chem., 78, 2128.

[9] Siomos, K. and Christophorou, L. G. (1980). Chem. Phys. Lett., 72, 13.

[10] Ogawa, T., Chen, H., Inoue, T. and Nakashima, K. (1994). Chem. Phys. Lett., 229, 328.

[11] Nakashima, K., Kise, M., Ogawa, T., Kawazumi, H. and Yamada, S. (1994). Chem. Phys. Lett., 231, 85.

[12] Birks, J., Dyson, D. J. and Murno, I. H. (1963). Proc. Roy. Soc., 275, 575.

[13] Schmidt, W. F. and Allen, A. O. (1968). J. Phys. Chem., 72, 3730.

[14] Boschi, R. and Schmidt, W. F. (1972). Tetrahedron Lett., 25, 2577.

[15] Siomos, K., Kourouklis, G. A. and Christophorou, L. G. (1981). Proc. 2nd International Swarm, seminar, 139.

[16] Katoh, R. and Kotani, M. (1993). Chem. Phys. Lett., 201, 141.

[17] Kourouklis, G. A., Siomos, K. and Christophorou, L. G. (1982). J. Mol. Spectrosc., 92, 127

[18] Masuhara, H., Miyasaka, H., Ikeda, N. and Mataga, N. (1981). Chem. Phys. Lett., 82, 59. 